Original paper

\title{
Continual improvement of nasopharyngeal carcinoma segmentation with less labeling effort
}

\author{
Kuo Men, Xinyuan Chen, Ji Zhu, Bining Yang, Ye Zhang, Junlin Yi, and Jianrong Dai " \\ National Cancer Center/National Clinical Research Center for Cancer/Cancer Hospital, Chinese Academy of Medical Sciences and Peking Union Medical College, Beijing \\ 100021, China
}

\section{A R T I C L E I N F O}

\section{Keywords:}

Radiotherapy

Automatic segmentation

Continual learning

Reduce labeling

\begin{abstract}
A B S T R A C T
Purpose: Convolutional neural networks (CNNs) offer a promising approach to automated segmentation. However, labeling contours on a large scale is laborious. Here we propose a method to improve segmentation continually with less labeling effort.

Methods: The cohort included 600 patients with nasopharyngeal carcinoma. The proposed method was comprised of four steps. First, an initial CNN model was trained from scratch to perform segmentation of the clinical target volume. Second, a binary classifier was trained using a secondary CNN to identify samples for which the initial model gave a dice similarity coefficient (DSC) $<0.85$. Third, the classifier was used to select such samples from the new coming data. Forth, the final model was fine-tuned from the initial model, using only selected samples. Results: The classifier can detect poor segmentation of the model with an accuracy of $92 \%$. The proposed segmentation method improved the DSC from 0.82 to 0.86 while reducing the labeling effort by $45 \%$.

Conclusions: The proposed method reduces the amount of labeled training data and improves segmentation by continually acquiring, fine-tuning, and transferring knowledge over long time spans.
\end{abstract}

\section{Introduction}

Segmentation of the target volumes (TVs) and organs-at-risk is a necessary step before treatment planning for radiotherapy. However, manual delineation is often very time-consuming and prone to inter- and intra-observer variation [1-3]. Convolutional neural networks (CNNs) offer a promising method to automatic segmentation [4-5]. Many studies report that CNNs have outstanding performance in segmentation for several disease types [6-10].

The performance of CNNs relies on large-scale annotated training samples. More good training samples are helpful to achieve better performance by covering wider data distribution. However, the amount of data is usually not large for the initial training of the network. With the accumulation of clinical data, more data are available to retrain the model. The models may deteriorate when training from scratch with new data, as previously learned knowledge is forgotten. Retraining with both old and new data could address catastrophic forgetting, but it is not convenient in the age of big data. First, as the training data increases, we need more storage for a stream of data. Second, it needs to combine all the data for each training session. The model should be able to continually learn over time from the increasing stream of medical information. This strategy is referred to as continual learning [11-12]. Although this concept has been proposed, few continual learning methods with CNNs are available for segmentation [13-14]. For example, Chan et al [14] reported a lifelong learning algorithm for automatic segmentation of head and neck organs. It's more like transfer learning and assumes all the data are available, which is not a continuous learning scenario.

Labeling large-scale contours is usually laborious and not necessarily feasible in real-world applications. Recently, there has been some work [15] to solve the problems of imperfect datasets. Shah et al [16] reduced the supervision cost by combining strong supervision with weak supervision. Mlynarski et al [17] extended the U-Net with an additional branch to exploit information contained in weakly-annotated images and ignore features which were irrelevant for the segmentation task. The most reliable approach to the scarce annotation problem is to obtain additional labeled examples [15]. Costs can be reduced by selecting the most appropriate unlabeled data to be labeled. Active learning [18-20] can achieve high quality performance using a limited labeled data set. Several query strategies [21] are available for active learning, including

\footnotetext{
* Corresponding author.

E-mail address: dai jianrong@cicams.ac.cn (Jianrong Dai).
} 

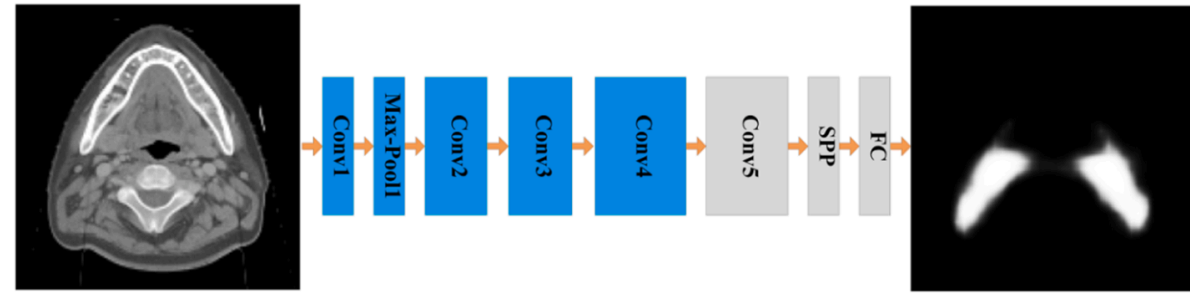

Fig. 1. Architecture of the segmentation network. The input to the CNN was the dataset of CT images, and the output was the corresponding segmentation probability map for the organ.

Table A1

Detailed architecture of the segmentation network.

\begin{tabular}{|c|c|c|}
\hline Layer & \multicolumn{2}{|c|}{ Convolution kernel } \\
\hline Input data & \multicolumn{2}{|l|}{ - } \\
\hline Conv1 & \multicolumn{2}{|l|}{$[7 \times 7,64]$} \\
\hline Max-Pool1 & \multicolumn{2}{|l|}{$[3 \times 3,64]$} \\
\hline Conv2 & \multicolumn{2}{|c|}{$\left[\begin{array}{c}1 \times 1,64 \\
3 \times 3,64 \\
1 \times 1,256\end{array}\right] \times 3$} \\
\hline Conv3 & {$\left[\begin{array}{l}1 \times 1,128 \\
3 \times 3,128 \\
1 \times 1,512\end{array}\right]$} & $\times 4$ \\
\hline Conv4 & {$\left[\begin{array}{c}1 \times 1,256 \\
3 \times 3,256 \\
1 \times 1,1024\end{array}\right.$} & $\times 23$ \\
\hline Conv4 & {$\left[\begin{array}{c}1 \times 1,512 \\
3 \times 3,512 \\
1 \times 1,2048\end{array}\right.$} & $\times 3$ \\
\hline SPP & $\begin{array}{l}{[1 \times 1,1024]} \\
{[3 \times 3,1024]} \\
{[3 \times 3,1024]} \\
{[3 \times 3,1024]}\end{array}$ & \\
\hline FC & {$[1 \times 1,2]$} & \\
\hline Interpolation & factor $=8$ & \\
\hline Output data & & \\
\hline
\end{tabular}

uncertainty sampling, query-by-committee, expected model change, expected error reduction, variance reduction, and density-weighted methods. The samples for which the current segmentation model performs poorly should be the most valuable for fine-tuning.

In this study, we propose a method to continually improve the segmentation with less labeling effort. The proposed method is novel in two of its main features. First, we introduced a binary classifier to predict the segmentation performance and to select the most useful samples to be labeled. Second, we revised a network to continually learn, fine-tune, and transfer knowledge throughout long time spans. To the best of our knowledge, this study is the first to decrease the labeling effort for sequential segmentation modeling and suggests an approach for training more robust segmentation models with continuous streams of information.

\section{Methods}

\subsection{Patient data}

A total of 600 patients with nasopharyngeal carcinoma that received radiotherapy were enrolled in this study. Patients were immobilized in the supine position with a thermoplastic mask (head, neck, shoulder). The simulation CT images were scanned on a Somatom Definition AS 40 (Siemens Healthcare, Forschheim, Germany) or a Brilliance CT Big Bore
(Philips Healthcare, Best, the Netherlands) with contrast enhancement and were reconstructed with a matrix of $512 \times 512$, a slice thickness of $3.0 \mathrm{~mm}$, and a pixel size of $0.96-1.27 \mathrm{~mm}$. The clinical target volume (CTV) was manually contoured directly on the CT with the aid of MR. Experienced radiation oncologists checked and confirmed all the clinical contours for quality control. There were about 200 2D CT slices per cases, about a third of which had contours. To avoid the false positive, all the slices with or without contours were included for model training in this study.

\subsection{The proposed continual learning framework}

The proposed continual learning framework includes three main components: a network to perform segmentation, a network to classify useful samples to be labeled, and a fine-tuning strategy for model retraining.

\subsubsection{The segmentation network}

A CNN with 103 convolutional layers [9] was trained to perform segmentation of the CTV in CT imaging. This network has been proven to have state-of-the-art performance for medical segmentation tasks. Fig. 1 illustrates the architecture of the segmentation network. The details of the architecture are presented in Table A1. The input was the CT image, and the output was the corresponding segmentation probability map for the CTV. It had 103 convolutional layers. In detail, the first convolution layer (Conv1) extracted 64 feature maps using a filter size of $7 \times 7$ and a stride of 2 . A max pooling with a filter size of $3 \times 3$ and a stride of 2 were used for down-sampling. Then multiple deeper bottleneck architectures (DBAs) [22] were used to ensure that gradients were not lost when the network became very deep. Each DBA consisted of three convolutional layers of $1 \times 1,3 \times 3$, and $1 \times 1$, where the $1 \times 1$ layers are responsible for reducing and then increasing dimensions, leaving the $3 \times 3$ layer a bottleneck with smaller input/output dimensions. There were 3 DBAs in Conv2, 4 DBAs in Conv3, 23 DBAs in Conv4, and 3 DBAs in Conv5, respectively. It was followed by a spatial pyramid pooling (SPP) module that consisted of four parallel convolution layers (one $1 \times 1$ filter and three $3 \times 3$ filters), which had different dilated rates $(1,4,8$, and 12 , respectively) and were added together. Finally, the fully connected layer (FC) was used for pixel-wise classification.

2.2.1.1. The classification network. Once the segmentation model was trained, its performance was evaluated using the Dice similarity coefficient (DSC). The DSC was used to compare the predicted segmentation against the manual contours (ground truth). It was calculated as DSC = $2 \mathrm{TP} /(2 \mathrm{TP}+\mathrm{FP}+\mathrm{FN})$ using the definition of true positive (TP), false positive (FP), and false negative (FN). It ranges from 0 , indicating no spatial overlap between the two segmentations, to 1 , indicating complete overlap. To improve the current model with new data, the samples on which the segmentation model performed poorly (low DSC) bear the most useful information for the current model to learn.

In this study, we used a DSC threshold of 0.85 to distinguish between good and poor segmentation quality. This threshold was established by 

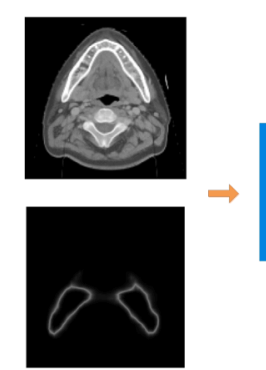

Binary Classifier \begin{tabular}{c}
$\begin{array}{c}\text { Select for re-training? } \\
0: \text { No } \\
1: Y e s\end{array}$ \\
\hline
\end{tabular}

Fig. 2. The binary classifier for sample selection. The input included the CT image and its corresponding uncertainty map. The output was whether this slice should be selected to be labeled for fine-tuning of the segmentation model. The threshold was set to DSC $<0.85$.

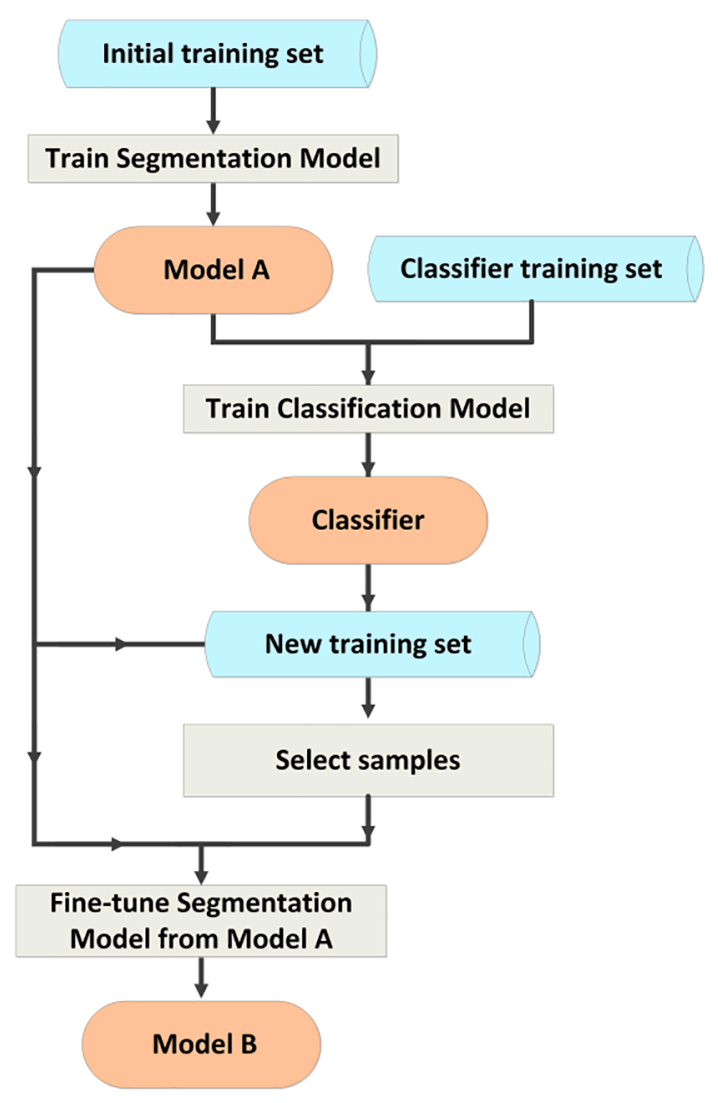

Fig. 3. Flowchart of the proposed method.

radiotherapy experts in our department based on clinical cases. In other words, radiotherapy experts concluded that automatic contours with DSC below 0.85 need to be significantly modified. We divided the samples into two categories: 0, the segmentation model performed well (DSC $\geq 0.85$ ); 1 , the segmentation did not work well (DSC $<0.85$ ).

More data is benefit to training a robust model. However, labeling on new imaging is time-consuming and laborious. If we want to improve the current model with the new coming training data, the samples on which the segmentation model performed poorly bear the most useful information for the current model to learn. But for these new cases without manual contours, we cannot evaluate the current model's performance on them. To reduce the labeling effort, we proposed a binary classifier that used a secondary CNN (ResNet-101) [22] to identify the samples categorized as 'poor' to be labeled. The segmentations with predicted DSC $>=0.85$ were qualified as "good" labels (i.e., the current model could segment well) and not included for retraining. The most useful samples on which the original segmentation model had DSC $<$
0.85 were selected for retraining.

We calculated the segmentation uncertainty map $(u(i, j))$ from the segmentation probability map $(p(i, j))$ according to formula 1 :

$u(i, j)=1-\max [p(i, j), 1-p(i, j)]$

The pixel values of segmentation uncertainty map ranged from 0 to 0.5 and the pixels closer to 0.5 correspond to those that lie close to the decision boundary of the segmentation model. That is to say, the segmentation uncertainty map represents the confidence of the segmentation model. This information relates closely to the quality of the automatic segmentation. In addition, the CT image is also helpful to judge the segmentation quality because it can provide information of patient geometry and image contrast. As shown in Fig. 2, the input of the classifier included the CT image and its corresponding segmentation uncertainty map. The output of the classifier was the prediction of segmentation quality, and those categorized as 'poor' were selected to be labeled for retraining.

\subsection{Model fine-tuning with new data}

If all the parameters of a network are re-trained with new data, the model may deteriorate as previously learned weights are updated. The convolutional layers at the front of CNNs extract basic features of the images such as shapes, intensity, and edges, while the deeper layers learn more complex features. To allow for continual learning of new knowledge while avoiding catastrophic forgetting, we locked most of the shallow layers (Conv1-Conv4) and only fine-tuned the last few layers (Conv5-FC), as shown in gray (Fig. 1). The old knowledge is retained in the shallow layers. The model parameters of unlocked layers were initialized using the weights from the old model.

\subsection{Experiments}

All 600 cases were divided into an initial training set (250 cases), a classifier training set ( 50 cases), a new training set (250 cases), and a test set (50 cases) in chronological order. Experienced radiation oncologists confirmed the manual contours of the 600 cases. The proposed method was comprised of four steps (Fig. 3). First, we trained Model A from scratch with the initial training set. Second, we applied Model A to the classifier training set to get the segmentation uncertainty map and DSC of each slice, and then trained and validated a binary classifier using a secondary CNN with the CT image and its corresponding segmentation uncertainty map as input, and DSC levels (threshold: 0.85) as output, respectively. This step was designed to identify samples from Model A that had a DSC below 0.85. Third, Model A was applied to the new training set to get the segmentation uncertainty maps and then the classifier was used to predict samples with predicted DSC $<0.85$ from the new training set. These slices with manually checked contours were selected for further fine-tuning. Finally, Model B was fine-tuned from Model A, using only the selected samples from the new training set.

To validate the proposed method, Model C was trained with both initial training and new training sets, Model D was trained from scratch with the new training set, and Model E was fine-tuned from model A using both the selected new samples and initial training set. We compared the performance of the five models (Model A through Model E) when applied to the test set.

To investigate the effect of dataset size on the performance of Model $\mathrm{B}$, we did an experiment that gradually increased the number of training samples. The model was fine-tuned from Model A gradually, using selected samples from 50 random patients in the new training set at a time.

Two CNNs were used in this study: a segmentation network and a classification network. All the training and testing were implemented on the Caffe platform using an NVIDIA TITAN XP GPU. Backpropagation with the stochastic gradient descent algorithm was used to optimize the layer weights. The learning rate policy was set to 'poly,' with a base 

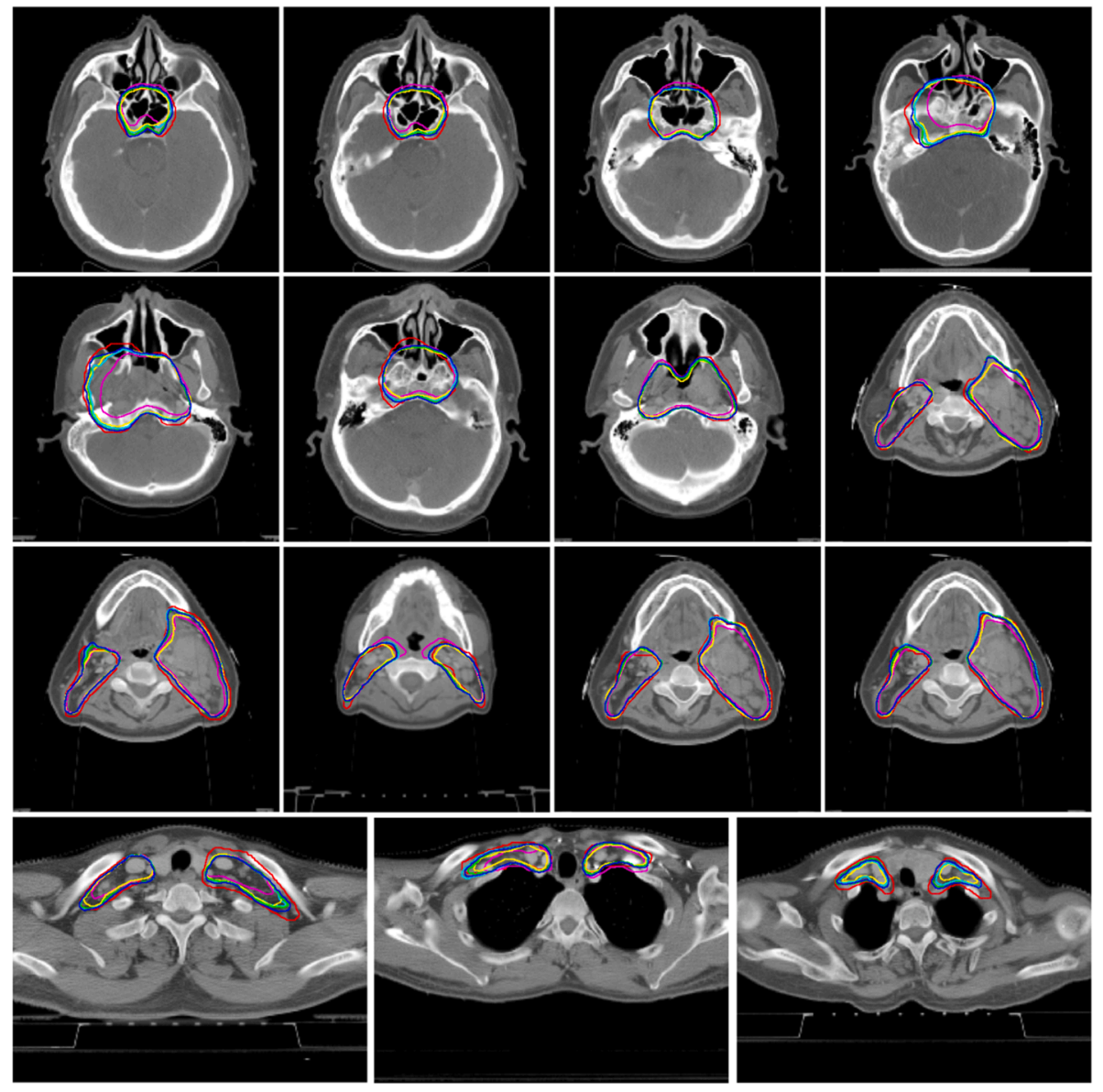

Fig. 4. Segmentation results for tested cases. Red, Ground Truth; Magenta, Model A; Blue, Model B (the proposed method); Green, Model C; Yellow, Model D; and Cyan, Model E. (For interpretation of the references to colour in this figure legend, the reader is referred to the web version of this article.)

learning rate of 0.001 and power of 0.9 . The momentum and weight decay were set to 0.9 and 0.0005 , respectively. The batch sizes were set to 1 for the segmentation network and 6 for the classification network, respectively. Some general methods, including random scaling, random cropping, and random rotation, were used for data augmentation.

\section{Results}

\subsection{Performance of the binary classifier}

The classifier was tested on the 250 cases from the new training set. The results show that the classifier can detect the poor segmentation (DSC $<0.85$ ) of the model with an accuracy of $92 \%$. The sensitivity, specificity, and the area under the receiving operator characteristic curve (AUC) of poor segmentation classification were $92 \%, 90 \%$ and 0.91 , respectively. With the classifier, only $55 \%$ slices from the new training set were selected to fine-tune the new model (Model B). In other words, the proposed method reduced $45 \%$ of the labeling effort.

\subsection{Performance of the segmentation model}

We trained five segmentation models to validate the proposed method. The application of Model A, Model B, Model C, Model D, and Model E to the test set resulted in DSCs of $0.82 \pm 0.03,0.86 \pm 0.02,0.86$ $\pm 0.02,0.83 \pm 0.03$, and $0.86 \pm 0.02$, respectively.

The segmentation models had low accuracy in the starting and ending slices due to the difficulty in extracting features and significant inter-observer variability. We analyzed the performance of the model on the 10 slices (including 5 slices at the beginning and 5 slices at the end) of the target boundary and the remaining middle part respectively. Model A had DSC values of 0.73 and 0.85 for the boundary and the middle part, respectively. Both Model B and C could improve DSC to 0.77 and 0.88 , respectively. Fig. 4 illustrates representative slices of the segmentation with Model A, the proposed Model B, Model C, Model D and Model E.

The method for training Model C, the most basic method of continual learning, involved putting all the data together and retraining. Model B was trained with the proposed continual learning. Both models had the best performance, with the DSC increasing from $0.82 / 0.83$ to 0.86 . The automated segmentation was in better agreement with the manual reference contour. These results demonstrate that the model allowed for continual learning of new knowledge while avoiding performance degradation.

Although the results show that the performance of Model B and Model C was comparable, the number of labeled samples for training Model B was $45 \%$ less than that for Model C. This finding proves that the method proposed in this study not only achieved continual learning but also screened out the most effective samples for training, thus reducing the amount of labeling work.

Model D, which was trained from scratch with the new training set, had comparable performance than Model A (trained with the old training set) and worse than the proposed Model $\mathrm{B}$. This is proved that the improvement of the Model B is not caused by the difference of training set data, but the proposed method.

Model E, which was fine-tuned from Model A with both the selected new samples and initial training set, also performed comparable with Model B and C. This proves that it's not necessary to add the previous data to the new data set with the proposed method. In other words, the 
proposed model can remember what it learned before.

When the number of training samples for Model B increased, the model performance was also improved correspondingly. The DSC was $0.83 \pm 0.03$ for 50 cases, $0.83 \pm 0.03$ for 100 cases, $0.85 \pm 0.03$ for 150 cases, $0.85 \pm 0.02$ for 200 cases, and $0.86 \pm 0.02$ for 250 cases, respectively. This proves that more data is useful to train a robust model and the proposed method can continuously learn new information.

\section{Discussion}

CNNs are increasingly used in radiotherapy for automatic segmentation and perform well over many disease sites. However, for supervised learning, CNNs need a large amount of labeled data to tune the free parameters. The labeling of sufficient contours is often very timeconsuming. In addition, CNNs may forget what was previously learned during retraining of the model with new data. This learning process differs from that of the human nervous system. The human brain can learn continually, accumulating large amounts of information. To the best of our knowledge, the present study is the first to combine continual learning and active learning for segmentation. The results show that the proposed method increased the DSC for segmentation of the CTV from 0.82 to 0.86 via lifelong learning and reduced the amount of labeling work by $45 \%$ via the active learning strategy. These findings demonstrate that the proposed method improves both the accuracy and efficiency of organ segmentation with CNNs.

While Model B and Model C performed comparably, Model C was trained from scratch using all the cases from both the training and new training sets. Although such retraining from scratch pragmatically avoids catastrophic forgetting, it is highly inefficient and hinders realtime learning of new data. The network for Model B locked the shallower layers that can retain old knowledge. Model B had fewer free parameters and lower training time, making this method more efficient and robust.

Two important features of our proposed method make it applicable to clinical practice. First, we introduced a quality classification network to predict the accuracy of the auto-segmentation generated by the current model. Only the samples that failed in the current model were labeled. This novel active learning method allows for the selection of the most useful information for further training, thereby greatly reducing the labeling effort. Second, we introduced a simple continual learning strategy that allowed for the acquisition of new information while retaining the old knowledge. The combination of these strategies not only reduces the amount of labeled training data but also improves segmentation by continually acquiring knowledge over long time spans.

The proposed method was evaluated on segmentation of the CTV. More organs need to be investigated in future investigations of this model. Another limitation is that we did not study the effect of the number of locked layers, instead locking a fixed number of layers. CNN models are more adjustable when fewer layers are locked but may lead to overfitting with too many parameters to tune. A comprehensive study will be performed to determine the optimal number of locked layers. In addition, we used a 2D rather than 3D segmentation model and counted the labeling reduction in terms of slices, because almost all of the patients had several slices with poor automatic segmentation.

\section{Conclusions}

Continual improvement of a segmentation model with minimal cost is particularly important in radiotherapy. Our proposed method decreases the amount of labeled training data and improves segmentation by continually acquiring, fine-tuning, and transferring knowledge over long time spans. This study provides guidance on how to train more robust segmentation models efficiently.

\section{Declaration of Competing Interest}

The authors declare that they have no known competing financial interests or personal relationships that could have appeared to influence the work reported in this paper.

\section{Acknowledgments}

This work was supported by the National Natural Science Foundation of China (11975313, 11875320), the Beijing Nova Program (Z201100006820058), the Beijing Municipal Science \& Technology Commission (Z181100001918002), and the Beijing Hope Run Special Fund of Cancer Foundation of China (LC2019B06, LC2018A14).

\section{References}

[1] Fiorino C, Reni M, Bolognesi A, et al. Intra-and inter-observer variability in contouring prostate and seminal vesicles: implications for conformal treatment planning. Radiother Oncol 1998;47(3):285-92.

[2] Li XA, Tai A, Arthur DW, et al. Variability of target and normal structure delineation for breast cancer radiotherapy: an RTOG Multi-Institutional and Multiobserver Study. Int J Radiat Oncol* Biol* Phys 2009;73(3):944-51.

[3] Eminowicz G, McCormack M. Variability of clinical target volume delineation for definitive radiotherapy in cervix cancer. Radiother Oncol 2015;117(3):542-7.

[4] Long J, Shelhamer E, Darrell T. Fully convolutional networks for semantic segmentation. In: Proceedings of the IEEE conference on computer vision and pattern recognition; 2015. p. 3431-40.

[5] Ronneberger O, Fischer P, U-net BT. Convolutional networks for biomedical image segmentation. In: International Conference on Medical image computing and computer-assisted intervention. Cham: Springer; 2015. p. 234-41.

[6] Ibragimov B, Xing L. Segmentation of organs-at-risks in head and neck CT images using convolutional neural networks. Med Phys 2017;44(2):547-57.

[7] Lustberg T, Soest JV, Gooding M, et al. Clinical evaluation of atlas and deep learning based automatic contouring for lung cancer. Radiother Oncol 2017;126 (2):312-7.

[8] Men K, Dai J, Li Y. Automatic segmentation of the clinical target volume and organs at risk in the planning CT for rectal cancer using deep dilated convolutional neural networks. Med Phys 2017;44(12):6377-89.

[9] Men K, Boimel P, Janopaul-Naylor J, et al. Cascaded atrous convolution and spatial pyramid pooling for more accurate tumor target segmentation for rectal cancer radiotherapy. Phys Med Biol 2018;63(18):185016.

[10] Charron O, Lallement A, Jarnet D, et al. Automatic detection and segmentation of brain metastases on multimodal MR images with a deep convolutional neural network. Comput Biol Med 2018:43-54.

[11] Parisi GI, Kemker R, Part JL, et al. Continual lifelong learning with neural networks: a review. Neural Networks 2019.

[12] Aljundi R, Punarjay C, Tinne T. Expert gate: lifelong learning with a network of experts. Proceedings of the IEEE Conference on Computer Vision and Pattern Recognition. 2017.

[13] Michieli U, Zanuttigh, P. Knowledge Distillation for Incremental Learning in Semantic Segmentation. ArXiv, 2019, abs/1911.03462.

[14] Chan JW, Kearney V, Haaf S, Wu, et al. A convolutional neural network algorithm for automatic segmentation of head and neck organs at risk using deep lifelong learning. Med Phys 2019;46(5):2204-13.

[15] Tajbakhsh N, Jeyaseelan L, Li Q, Chiang JN, Wu Z, Ding X. Embracing imperfect datasets: a review of deep learning solutions for medical image segmentation. Med Image Anal 2020;101693.

[16] Shah MP, Merchant SN, Awate SP. MS-Net: mixed-supervision fully-convolutional networks for full-resolution segmentation. In: International Conference on Medical Image Computing and Computer-Assisted Intervention. Cham: Springer; 2018. p. $379-87$.

[17] Mlynarski P, Delingette H, Criminisi A, Ayache N. Deep learning with mixed supervision for brain tumor segmentation. J Med Imaging 2019;6(3):034002.

[18] Dutt Jain S, Grauman K. Active image segmentation propagation[C]//Proceedings of the. IEEE Conference on Computer Vision and Pattern Recognition. 2016:: 2864-73.

[19] Yang L, Zhang Y, Chen J, et al. Suggestive annotation: A deep active learning framework for biomedical image segmentation[C]//International Conference on Medical Image Computing and Computer-Assisted Intervention. Cham: Springer; 2017. p. 399-407.

[20] Folmsbee J, Liu X, Brandwein-Weber M, et al. Active deep learning: Improved training efficiency of convolutional neural networks for tissue classification in oral cavity cancer. 2018 IEEE 15th International Symposium on Biomedical Imaging (ISBI 2018). IEEE; 2018.

[21] Settles B, Craven M. An Analysis of Active Learning Strategies for Sequence Labeling Tasks[C]//2008 Conference on Empirical Methods in Natural Language Processing, EMNLP 2008, Proceedings of the Conference, 25-27 October 2008, Honolulu, Hawaii, USA, A meeting of SIGDAT, a Special Interest Group of the ACL. Association for Computational Linguistics; 2008.

[22] He K, Zhang X, Ren S, et al. Deep residual learning for image recognition. 2016 IEEE Conference on Computer Vision and Pattern Recognition (CVPR). 2016. 